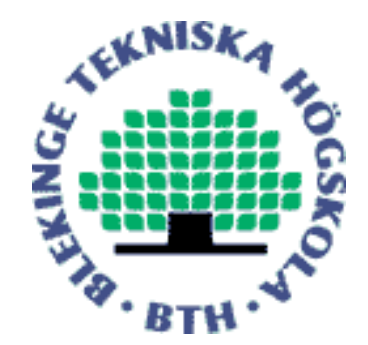

Copyright @ 2013 IEEE.

Citation for the published paper:

Title:

Phase Error Calculation for Fast Time-Domain Bistatic SAR Algorithms

Author:

Viet Thuy Vu, Thomas Sjögren, Mats Pettersson

Journal:

IEEE transactions on aerospace and electronic systems

Year:

2013

Vol:

49

Issue:

1

Pagination:

631-639

URL/DOI to the paper:

10.1109/TAES.2013.6404127

This material is posted here with permission of the IEEE. Such permission of the IEEE does not in any way imply IEEE endorsement of any of BTH's products or services Internal or personal use of this material is permitted. However, permission to reprint/republish this material for advertising or promotional purposes or for creating new collective works for resale or redistribution must be obtained from the IEEE by sending a blank email message to pubs-permissions@iee.org.

By choosing to view this document, you agree to all provisions of the copyright laws protecting it. 
Track segment association for GMTI tracks of evasive move-stop-move maneuvering targets.

IEEE Transactions on Aerospace and Electronic Systems, 47, 3 (July 2011), 1899-1914.

[14] Kirubarajan, T. and Bar-Shalom, Y.

Tracking evasive move-stop-move maneuvering targets with a GMTI radar using a VS-IMM estimator.

IEEE Transactions on Aerospace and Electronic Systems, 39, 3 (July 2003), 1098-1103.

[15] Lin, L., Bar-Shalom, Y., and Kirubarajan, T.

New assignment-based data association for tracking move-stop-move targets.

IEEE Transactions on Aerospace and Electronic Systems, 40, 2 (Apr. 2004), 714-725.

[16] Chong, C-Y., et al.

Efficient multiple hypothesis tracking by track segment graph.

Proceedings of the 12th International Conference on Information Fusion, (Fusion 2009), Seattle, WA, July 6-9, 2009, pp. 2177-2184.

[17] Castañón, G. and Finn, L.

Multi-target tracklet stitching through network flows.

Proceedings of the IEEE Aerospace Conference, Big Sky, MT, Mar. 2011, pp. 1-7.

[18] Song, T. L. and Mušicki, D.

Adaptive clutter measurement density estimation for improved target tracking.

IEEE Transactions on Aerospace and Electronic Systems, 47, 2 (Apr. 2011), 1457-1466.

[19] Bar-Shalom, Y., Li, X-R., and Kirubarajan, T. Estimation with Application to Tracking and Navigation. Hoboken, NJ: Wiley, 2001.

[20] Blackman, S.

Multiple-Target Tracking with Radar Applications.

Norwood, MA: Artech House, 1986.

[21] Salmond, D. J.

Mixture reduction algorithms for target tracking in clutter. Proceedings of SPIE, Signal and Data Processing of Small Targets, vol. 1305, Orlando, FL, Apr. 1990, pp. 434-445.

[22] Williams, J. L. and Mayback, P. S.

Cost-function-based Gaussian mixture reduction for target tracking.

Proceedings of the 6th International Conference on Information Fusion, (Fusion 2003), Cairns, Queensland, Australia, July 2003, pp. 1047-1054.

[23] Singer, R. A., Sea, R. G., and Housewright, K.

Derivation and evaluation of improved tracking filters for use in dense multi-target environments.

IEEE Transactions on Information Theory, IT-20, 4 (July 1974), 423-432.

[24] Bar-Shalom, Y., Chang, K., and Blom, H.

Automatic track formation in clutter with a recursive algorithm.

Multitarget Multisensor Tracking, vol. 1, Norwood, MA: Artech House, 1990, pp. 25-42.

\section{Phase Error Calculation for Fast Time-Domain Bistatic SAR Algorithms}

In synthetic aperture radar (SAR) processing, there is a trade-off between accuracy and speed. The approximations in an algorithm help to increase the algorithm's speed but cause deterministic phase errors which directly affect the SAR image quality. This paper discusses the phase error calculations for bistatic fast backprojection (BiFBP) and bistatic fast factorized backprojection (BiFFBP) which are essential for setting their parameters. The phase error calculation principle for bistatic SAR in comparison to monostatic SAR is presented. This principle is used to derive the maximum phase error equation.

\section{LIST OF SYMBOLS}

$R_{t} \quad$ True travel distance of radar pulse to TX

$R_{r} \quad$ True travel distance of radar pulse to RX

$R \quad$ True travel distance of radar pulse

$R_{t}^{\prime} \quad$ Processing travel distance of radar pulse to TX

$R_{r}^{\prime} \quad$ Processing travel distance of radar pulse to $\mathrm{RX}$

$R^{\prime} \quad$ Processing travel distance of radar pulse

$2 \alpha \quad$ Angle between $\vec{R}_{t}$ and $\vec{R}_{r}$

$\Delta r \quad$ Deterministic range error

$\Delta \phi \quad$ Deterministic phase error

$\epsilon_{t} \quad$ Length in calculation for transmitter subaperture

$\epsilon_{r} \quad$ Length in calculation for receiver subaperture

$\epsilon_{i} \quad$ Size in calculation for subimage

$\vec{\sigma}_{t} \quad$ First auxiliary vector on transmitter side

$\vec{\sigma}_{r} \quad$ First auxiliary vector on receiver side

$\vec{\rho}_{t} \quad$ Second auxiliary vector on transmitter side

$\vec{\rho}_{r} \quad$ Second auxiliary vector on receiver side

$\beta_{t} \quad$ Angle between transmitter flight track and $\vec{\sigma}_{t}$

$\Delta \beta_{t} \quad$ Angle between $\vec{\sigma}_{t}$ and $\vec{\rho}_{t}$

$\beta_{r} \quad$ Angle between receiver flight track and $\vec{\sigma}_{r}$

$\Delta \beta_{r} \quad$ Angle between $\vec{\sigma}_{r}$ and $\vec{\rho}_{r}$.

\section{INTRODUCTION}

Bistatic synthetic aperture radar (SAR) plays an important role in geoscience and remote sensing applications. Such applications are of interest to both military and civilian end-users. This can be shown by

Manuscript received June 27, 2011; revised November 16, 2011; released for publication January 25, 2012.

IEEE Log No. T-AES/49/1/944377.

Refereeing of this contribution was handled by R. Narayanan.

This work was supported by the KK-Foundation.

0018-9251/13/\$26.00 (c) 2013 IEEE 
a large number of publications about bistatic SAR. For example, a general processing approach for bistatic SAR is suggested in [1]. The approach allows solving problems caused by the lack of precise time (range) and phase (Doppler) references in noncooperative (and even cooperative) bistatic systems. A SAR data simulator for bistatic SAR of a translational invariant case, which is seen as an important tool for testing the system parameters, is introduced in [2]. In another publication [3] the ability to detect targets in a background of forest and urban clutter is shown to be enhanced significantly by a suitable selection of bistatic SAR geometry in comparison to monostatic SAR.

Bistatic SAR systems are supposed to be more resistant to RF jammers than monostatic SAR systems. SAR processing requires much computation capacity and that task may only be available at ground stations. This can be easily handled with the flexibility in deploying receiver(s) of bistatic SAR systems. With the multiple deployed receivers of bistatic SAR systems, an object can be observed at different angles. This enhances the classification ability of that object as well as giving tomographic capacities [4]. From a system designer's point of view, designing bistatic SAR is more flexible than monostatic SAR. A number of bistatic SAR systems have been built recently. The $\mathrm{X}$-band spaceborne-airborne bistatic SAR employing TerraSAR-X as transmitter and F-SAR as receiver [5], SABRINA with a ground-based C-band receiver and transmitters mounted on ESA ERS-2 and ENVISAT [6] are typical examples of experimental bistatic SAR systems.

Reconstructing SAR scenes from bistatic SAR echoes is handled by bistatic SAR algorithms. Similar to the monostatic SAR algorithms, the bistatic SAR algorithms are categorized in two groups: frequency- and time-domain. The mostly used bistatic frequency-domain algorithms are range Doppler [7, 8], range migration $[9,10]$, and nonlinear chirp scaling [11], which are seen to be extended versions of the corresponding monostatic frequency-domain algorithms. However, most of the bistatic frequency-domain algorithms only work under special circumstances, for example, the range Doppler algorithm for bistatic is available for azimuth-invariant bistatic configurations, i.e., the flight tracks of the platforms are parallel and the platform velocities are identical. The same circumstances are also required by the bistatic version of range migration. Such requirements are not always satisfied in reality. Nonlinear chirp scaling extended for bistatic cases is declared to have the ability to handle different bistatic SAR configurations. There are some approximations in chirp scaling in general and the bistatic version in particular may cause large phase errors. Also, chirp scaling is valid for a limited number of SAR systems like narrowband-narrowbeam (NB) SAR systems. For complicated bistatic configurations, ultrawideband-ultrawidebeam (UWB) cases, and if minimum phase error is required, processing SAR echoes must rely on bistatic time-domain SAR algorithms. Bistatic global backprojection (BiGBP) [12], bistatic fast backprojection (BiFBP) [12], and bistatic fast factorized backprojection (BiFFBP) $[13,14]$ all work in time-domain and have shown good performance with bistatic SAR configurations, especially for UWB SAR systems. The typical characteristics such as unlimited scene size, real time processing, local processing, manageable motion compensation, and large range migration handling are seen to be general advantages of time-domain algorithms over frequency-domain algorithms. In addition, BiGBP, BiFBP, and BiFFBP are not limited by any configuration of bistatic SAR, i.e., there is no limit for speed, flight track, and flight altitude of transmitter and receiver platforms.

Among the bistatic time-domain SAR algorithms, BiGBP is the basic for all other algorithms. Since there is no approximation made in BiGBP, it does not cause any phase error though it requires an extremely high computational burden. This shortcoming is easily overcome with the fast time-domain SAR algorithms BiFBP and BiFFBP with acceptable phase errors. Calculating phase errors caused by approximations made in BiFBP and BiFFBP is essential and obviously helps us to set the processing parameters in these algorithms.

The objective of this paper is to introduce a phase error calculation principle for bistatic time-domain SAR algorithms. The differences between the phase error calculations for monostatic and for bistatic SAR are therefore addressed. The principle of calculating phase error for bistatic is then applied to the phase error calculations for the fast time-domain bistatic SAR algorithms BiFBP and BiFFBP.

The paper is organized as follows. Section II introduces a phase error calculation principle for bistatic time-domain SAR algorithms. The phase error equation for the fast time-domain bistatic SAR algorithms BiFBP and BiFFBP is derived in Section III. Simulation results and validations are presented in Section IV. Section V provides the conclusions.

\section{PRINCIPLES OF CALCULATING PHASE ERROR}

Let us first consider a monostatic SAR system as shown in Fig. 1 and assume that there exists an object on the ground scene at the coordinate $\left(x_{m}, y_{n}\right)$. The true two-way travel distance of a radar pulse between an aperture position and this object is determined by

$$
R=2 \sqrt{\left(v_{p l} t-x_{m}\right)^{2}+y_{n}^{2}+h^{2}}
$$




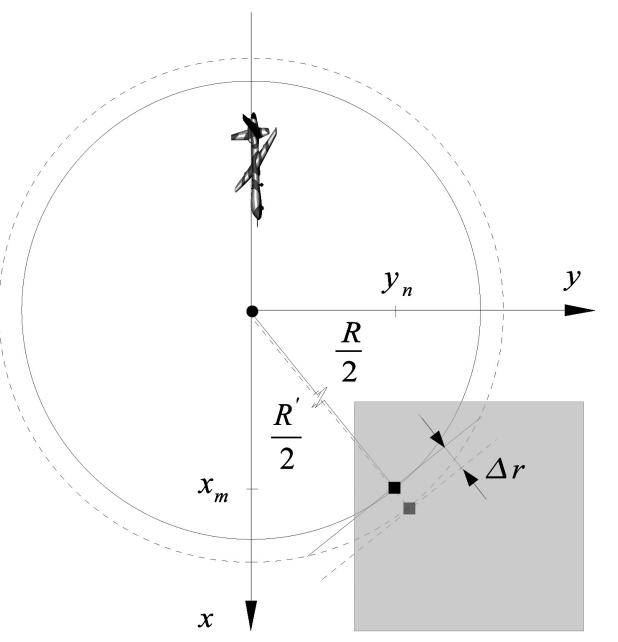

Fig. 1. Principles of calculating phase error for monostatic SAR. Monostatic radar is placed in center of coordinate system whereas ground object is located at coordinate $\left(x_{m}, y_{n}\right)$.

where $v_{p l}$ is the speed of the platform, $t$ is slow time (azimuth-time), and $h$ indicates the flight altitude. The true range between the aperture position and the object is given by $R / 2$ as shown in Fig. 1 .

Let us assume that due to approximations in processing, the processed two-way travel distance is now given by $R^{\prime}$ instead of $R$. This difference $\Delta R=R^{\prime}-R$ causes a range error $\Delta r$ in a SAR image. On a spherical basis applied to monostatic processing, the range error is the space between two tangents to the corresponding circles and is defined by

$$
\Delta r=\frac{1}{2} \Delta R=\frac{1}{2}\left(R-R^{\prime}\right) .
$$

The phase error is then estimated from range error $\Delta r$ by

$$
\Delta \phi=k_{c} \Delta r=\frac{k_{c}}{2}\left(R-R^{\prime}\right)
$$

where $k_{c}$ is the wavenumber associated with the center frequency $f_{c}$ of the radar signal.

The same SAR scene is now assumed to be illuminated by a bistatic SAR system as illustrated in Fig. 2. The true travel distance of a radar pulse from a transmitter aperture position impinging on the object on the ground scene $\left(x_{m}, y_{n}\right)$, then scattering, and finally approaching a receiver aperture position is shown to be

$$
\begin{aligned}
R= & R_{t}+R_{r} \\
= & \sqrt{\left(v_{t, x} t-x_{m}\right)^{2}+\left(v_{t, y} t-y_{n}\right)^{2}+h_{t}^{2}} \\
& +\sqrt{\left(v_{r, x} t-x_{m}\right)^{2}+\left(v_{r, y} t-y_{n}\right)^{2}+h_{r}^{2}}
\end{aligned}
$$

where $v_{t}$ and $v_{r}$ denote the speed of the transmitter and receiver platforms, respectively. The subscripts $x$ and $y$ indicate the speed components. The flight

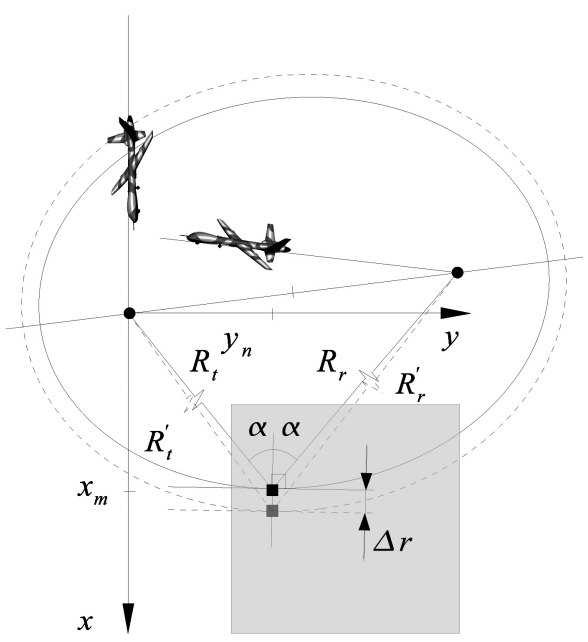

Fig. 2. Principles of calculating phase error for bistatic SAR. Transmitter is located at circular black point and receiver at other black point. Object has same coordinate as in Fig. 1.

altitudes of the transmitter and the receiver are denoted by $h_{t}$ and $h_{r}$, respectively.

The processed travel distance is assumed to be $R^{\prime}=R_{t}^{\prime}+R_{r}^{\prime}$ also causing a range error $\Delta r$. On an ellipsoidal basis applied to bistatic processing, the range error is the space between two tangents to the corresponding ellipses. Since the space between these two concentric ellipses is usually very small in comparison to the travel distances of a radar pulse, i.e., $\Delta r \ll R_{t}$ and $\Delta r \ll R_{r}$, these tangents are almost parallel. Based on the characteristics of a triangle, the relationships between $R_{t}$ and $R_{t}^{\prime}$ can be expressed by the law of cosine as

$$
\begin{aligned}
R_{t}^{\prime} & =\sqrt{R_{t}^{2}+\Delta r^{2}-2 R_{t} \Delta r \cos (\pi-\alpha)} \\
& =R_{t} \sqrt{1+\left(\frac{\Delta r}{R_{t}}\right)^{2}+2\left(\frac{\Delta r}{R_{t}}\right) \cos \alpha}
\end{aligned}
$$

where $\alpha$ is half of the angle between the generator lines $R_{t}$ and $R_{r}$. In other words the perpendicular to the tangent through the contact point bisects the angle $2 \alpha$. The square root term on the right hand side of (5) has the form of $\sqrt{1+X}$ and this function can therefore be expanded using the Taylor series as follows

$$
\begin{aligned}
R_{t}^{\prime}=R_{t}\{1 & +\frac{1}{2}\left[2\left(\frac{\Delta r}{R_{t}}\right) \cos \alpha+\left(\frac{\Delta r}{R_{t}}\right)^{2}\right]^{1} \\
& \left.-\frac{1}{8}\left[2\left(\frac{\Delta r}{R_{t}}\right) \cos \alpha+\left(\frac{\Delta r}{R_{t}}\right)^{2}\right]^{2}+\cdots\right\} \\
= & R_{t}+\Delta r \cos \alpha+\frac{\Delta r^{2}-\Delta r^{2} \cos ^{2} \alpha}{2 R_{t}}+\cdots .
\end{aligned}
$$

Due to the fact that $\Delta r \ll R_{t}$, the terms excepted the two first terms of (6) are approximated to zeros. 
Hence, $R_{t}^{\prime}$ is approximately given by

$$
R_{t}^{\prime} \approx R_{t}+\Delta r \cos \alpha .
$$

In a similar way, the following approximation is achieved for $R_{r}$

$$
R_{r}^{\prime} \approx R_{r}+\Delta r \cos \alpha
$$

Adding the left and right hand sides of (7) to those of (8) results in

$$
\begin{aligned}
R_{t}^{\prime}+R_{r}^{\prime} & \approx R_{t}+R_{r}+2 \Delta r \cos \alpha \\
R^{\prime} & \approx R+2 \Delta r \cos \alpha .
\end{aligned}
$$

The range error in processing for bistatic SAR is therefore defined by

$$
\Delta r \approx \frac{1}{2 \cos \alpha}\left(R^{\prime}-R\right) .
$$

The phase error for bistatic SAR is then retrieved with (3) and given by

$$
\Delta \phi=\frac{k_{c}}{2 \cos \alpha}\left(R^{\prime}-R\right)
$$

Generally, the phase error changes with respect to the positions of platforms and of a SAR scene, or in other words $\alpha$. According to (11), the minimum value of $\cos \alpha$ will maximize the phase error $\Delta \phi$. For this reason, $\cos \alpha$ should be calculated with the extreme positions of platforms and of SAR scenes in order to find the maximum phase error. To simplify calculations, we suppose that for small and medium SAR scenes, an angle $\alpha$ can be calculated with the aim point of that SAR scene.

\section{PHASE ERROR EQUATION FOR FAST TIME-DOMAIN BISTATIC SAR ALGORITHMS}

BiFBP [12] is one of the fast time-domain bistatic SAR algorithms which have been developed recently. BiFBP processes SAR echoes on a subaperture and subimage basis. This means that the full reconstructed scene is segmented into a number of subimages while the complete transmitter and receiver apertures are split into a number of subapertures. The reconstruction of the imaged scene is processed in two stages: beamforming and backprojection. Figure 3 shows an example in which a subaperture is formed with four aperture positions and a subimage is one-fourth of the full reconstructed SAR scene. The mathematical expression of BiFBP is given by

$$
h\left(x_{m}, y_{n}\right)=\bigcup_{k=1}^{K} \sum_{l=1}^{L} \int_{t_{l}-\left(t_{s} / 2\right)}^{t_{l}+\left(t_{s} / 2\right)} g\left(t, \frac{R_{m, n}^{c}-R_{l, k}^{c}+R_{l, k}(t)}{\mathrm{c}}\right) d t
$$

where $K$ is the number of subimages, $L$ is the number of subapertures, $t_{l}$ is the time when the platforms are at the centers of the $l$ th subapertures, and $t_{s}$ is the integration time. The range compressed radar echo

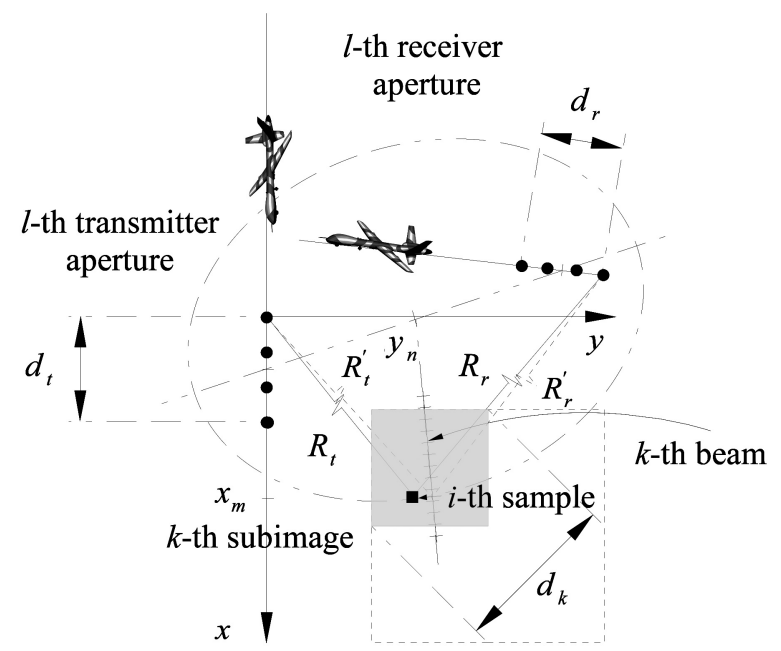

Fig. 3. Range error analysis in BiFBP. Solid lines indicate true travel distance of radar pulse while dashed lines indicate processed travel distance. Dotted-dashed ellipse shows ellipsoidal mapping.

is represented by $g(t, \tau)$ which is a function of both the azimuth (slow) time $t$ and the range (fast) time $\tau$. The distance from an aperture position belonging to the $l$ th transmitter subaperture via the center of the $k$ th subimage to the corresponding aperture position belonging to the $l$ th receiver subaperture is given by $R_{l, k}(t)$ and depends on the azimuth time $t$. The total distance between the center of the $l$ th transmitter subaperture and the center of the $l$ th receiver subaperture via the center of the $k$ th subimage is denoted by $R_{l, k}^{c}$. $R_{m, n}^{c}$ is the total distance between the center of the $l$ th transmitter subaperture and the center of the $l$ th receiver subaperture via the image pixel $\left(x_{m}, y_{n}\right)$ belonging to the $k$ th subimage.

Let us consider one of the transmitter aperture positions belonging to the $l$ th transmitter subaperture, e.g. the first one, the image pixel $\left(x_{m}, y_{n}\right)$, and the corresponding receiver aperture position belonging to the $l$ th receiver subaperture, i.e., also the first one. The true travel distance of a radar pulse in this consideration is given by $R=R_{t}+R_{r}$ and marked by the solid lines in Fig. 3.

In the beamforming stage of BiFBP, the $k$ th beam is formed by first shifting all radar echoes belonging to the $l$ th subapertures with respect to the center of the $k$ th subimage, i.e., $R_{l, k}(t)$, and then superposing them. In the backprojection stage of BiFBP, the image pixel $\left(x_{m}, y_{n}\right)$ belonging to the $k$ th subimage is actually mapped by the $i$ th sample of the $k$ th beam, which is found from $R_{m, n}^{c}-R_{l, k}^{c}$, over an ellipsoidal mapping. The mapping is marked by a dotted-dashed ellipse in Fig. 3 whose major axis is the line connecting the center of the $l$ th subapertures. The processed travel distance is therefore nothing else but the distance from the considered transmitter aperture position via the $i$ th sample of the $k$ th beam to the considered receiver aperture position, i.e., $R^{\prime}=R_{t}^{\prime}+R_{r}^{\prime}$. The processed 


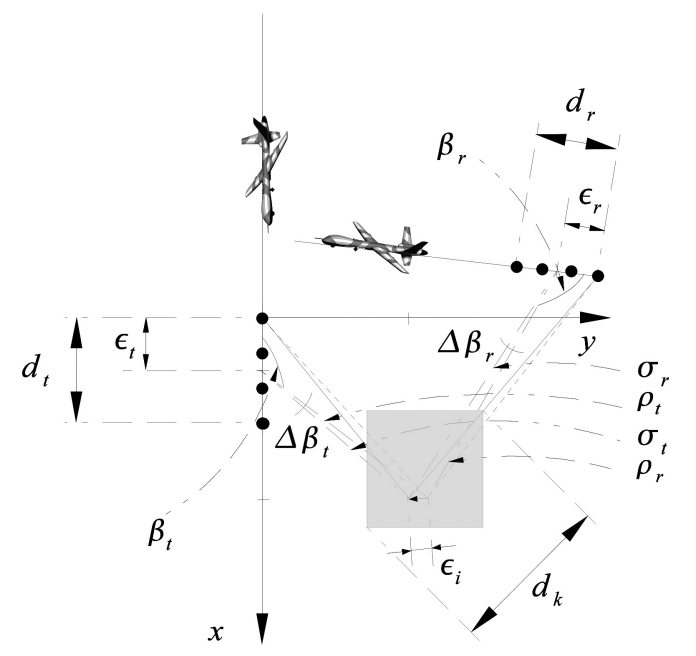

Fig. 4. Range error analysis in BiFBP (cont.). Symbols used in range error analysis are summarized in the list of symbols and physically explained in the derivations. Distances $R_{t}, R_{t}^{\prime}, R_{r}$, and $R_{r}^{\prime}$ in this figure are identical to the ones in Fig. 3 .

distance is marked by the dashed lines in Fig. 3. The difference between the true travel distance $R$ and the processed travel distance $R^{\prime}$ causes a range error and therefore a phase error in the reconstructed SAR scene.

Let $\epsilon_{t}$ be the space between the center of a transmitter subaperture and an arbitrary aperture position belonging to that subaperture. This space is obviously not more than half of the subaperture length. Similarly, we use $\epsilon_{r}$ to denote the distance from the center of a receiver subaperture to an arbitrary aperture position belonging to the considered receiver subaperture. The example illustrated in Fig. 4 corresponds to the maximum values of $\epsilon_{t}$ and $\epsilon_{r}$. Auxiliary vectors are interpreted as the vectors which are temporarily used in the following derivation. In this case the auxiliary vectors $\vec{\sigma}_{t}, \vec{\sigma}_{r}, \vec{\rho}_{t}$, and $\vec{\rho}_{r}$ are marked by the dotted-dashed lines in Fig. 4 and used to formed different triangles which allow us to apply the law of cosine. On the transmitter side the flight track and $\vec{\sigma}_{t}$ form the angle $\beta_{t}$ whereas $\Delta \beta_{t}$ is the angle between $\vec{\sigma}_{t}$ and $\vec{\rho}_{t}$. On the receiver side $\beta_{r}$ is formed by the flight track of the receiver platform and $\vec{\sigma}_{r}$, whereas the angle between $\vec{\sigma}_{r}$ and $\vec{\rho}_{r}$ is $\Delta \beta_{r}$.

Based on the auxiliary vectors $\vec{\sigma}_{t}, \vec{\sigma}_{r}$ and the angles $\beta_{t}, \Delta \beta_{t}, \beta_{r}, \Delta \beta_{r}$, the true travel distance of a radar pulse is determined by the law of cosine as

$$
\begin{aligned}
R= & R_{t}+R_{r} \\
= & \sqrt{\sigma_{t}^{2}+\epsilon_{t}^{2}-2 \sigma_{t} \epsilon_{t} \cos \left(\beta_{t}+\Delta \beta_{t}\right)} \\
& +\sqrt{\sigma_{r}^{2}+\epsilon_{r}^{2}-2 \sigma_{r} \epsilon_{r} \cos \left(\beta_{r}+\Delta \beta_{r}\right)} .
\end{aligned}
$$

Applying the Taylor expansion for the square root terms on the right hand side of (13) and taking only the first two terms of the Taylor series into account, the true travel distance is then approximated by

$$
R \approx\left(\sigma_{t}+\sigma_{r}\right)-\epsilon_{t} \cos \left(\beta_{t}+\Delta \beta_{t}\right)-\epsilon_{r} \cos \left(\beta_{r}+\Delta \beta_{r}\right) .
$$

Similarly, based on the auxiliary vectors $\vec{\rho}_{t}, \vec{\rho}_{r}$ and the angles $\beta_{t}, \beta_{r}$, the processed travel distance is shown to be

$$
\begin{aligned}
R^{\prime} & =R_{t}^{\prime}+R_{r}^{\prime} \\
& =\sqrt{\rho_{t}^{2}+\epsilon_{t}^{2}-2 \sigma_{t} \epsilon_{t} \cos \beta_{t}}+\sqrt{\rho_{r}^{2}+\epsilon_{r}^{2}-2 \sigma_{r} \epsilon_{r} \cos \beta_{r}}
\end{aligned}
$$

and then approximated by

$$
R^{\prime} \approx\left(\rho_{t}+\rho_{r}\right)-\epsilon_{t} \cos \beta_{t}-\epsilon_{r} \cos \beta_{r} .
$$

According to the characteristics of an ellipse, the following hold true

$$
\sigma_{t}+\sigma_{r}=\rho_{t}+\rho_{r} .
$$

In BiFBP the processed travel distance is approximated to the true travel distance of a radar pulse, i.e., $R^{\prime} \approx R$. Using (11) and taking (17) into account, the range error caused by this approximation is calculated as follows

$$
\begin{aligned}
\Delta r= & \frac{1}{2 \cos \alpha}\left(R^{\prime}-R\right) \\
= & \frac{\epsilon_{t}}{2 \cos \alpha}\left[\cos \left(\beta_{t}+\Delta \beta_{t}\right)-\cos \beta_{t}\right] \\
& +\frac{\epsilon_{r}}{2 \cos \alpha}\left[\cos \left(\beta_{r}+\Delta \beta_{r}\right)-\cos \beta_{r}\right] \\
= & \frac{\epsilon_{t}}{\cos \alpha} \sin \left(\frac{\Delta \beta_{t}}{2}\right) \sin \left(\beta_{t}+\frac{\Delta \beta_{t}}{2}\right) \\
& +\frac{\epsilon_{r}}{\cos \alpha} \sin \left(\frac{\Delta \beta_{r}}{2}\right) \sin \left(\beta_{r}+\frac{\Delta \beta_{r}}{2}\right) .
\end{aligned}
$$

As mentioned the values of $\epsilon_{t}$ and $\epsilon_{r}$ cannot be beyond the ranges $0 \leq \epsilon_{t} \leq d_{t} / 2$ and $0 \leq \epsilon_{r} \leq d_{r} / 2$, respectively, where $d_{t}$ and $d_{r}$ are the subaperture lengths.

Let $\epsilon_{i}$ be the space between a beam sample and an image pixel ellipsoidaly mapped by that beam sample. Since the maximum value of $\epsilon_{i}$ is given by $d_{k} / 2$ where $d_{k}$ is maximum dimension of a subimage, i.e., the diagonal, and $\Delta \beta_{t}$ is very small, the maximum of the first sin function in (18) is approximately given by

$$
\max \left\{\sin \left(\frac{\Delta \beta_{t}}{2}\right)\right\} \approx \max \left\{\frac{\epsilon_{i}}{2 r_{t}}\right\}=\frac{d_{k}}{4 r_{t, 0}}
$$

where $r_{t}$ is the distance from the center of a transmitter subaperture to an image pixel and $r_{t, 0}$ denotes the minimum range value. The maximum of the second sin function in (18) is simply determined by

$$
\max \left\{\sin \left(\beta_{t}+\frac{\Delta \beta_{t}}{2}\right)\right\}=1
$$


TABLE I

CARABAS-II and LORA Parameters

\begin{tabular}{ccc}
\hline \hline Parameter & $\begin{array}{c}\text { CARABAS-II } \\
\text { (transmitter) }\end{array}$ & $\begin{array}{c}\text { LORA } \\
\text { (receiver) }\end{array}$ \\
\hline The highest frequency processed $f_{\max }$ & $82.5 \mathrm{MHz}$ & \\
The lowest frequency processed $f_{\min }$ & $21.9 \mathrm{MHz}$ & $130 \mathrm{~m} / \mathrm{s}$ \\
Platform speed $v_{p l}$ & $126 \mathrm{~m} / \mathrm{s}$ & 4096 \\
Number of aperture positions $N_{l}$ & 4096 & $0.9673 \mathrm{~m}$ \\
Aperture step & $0.9375 \mathrm{~m}$ & $2900 \mathrm{~m}$ \\
Flight altitude & $3700 \mathrm{~m}$ & $3000 \mathrm{~m}$ \\
Minimum range $r_{0}$ & $5900 \mathrm{~m}$ & \\
Pulse repetition frequency & $137 \mathrm{~Hz}$ & $60^{\circ}$ \\
Angle formed by the flight tracks & & \\
\hline
\end{tabular}

when $\beta_{t}+\Delta \beta_{t} / 2=90^{\circ}$. Similar extrema can be derived for the third and fourth sin functions in (18). The upper limit of the range error is finally defined by

$$
\Delta r \leq \frac{d_{k}}{8 \cos \alpha}\left(\frac{d_{t}}{r_{t, 0}}+\frac{d_{r}}{r_{r, 0}}\right)
$$

In some special cases where the minimum ranges for both transmitter and receiver are the same ranges $r_{t, 0}=r_{r, 0}=r_{0}$, the platforms have the same speeds, i.e., $d_{t}=d_{r}=d_{l}$, and these platforms are in alignment, i.e., $\alpha=0^{\circ}$. The maximum range error equation (21) can be simplified to

$$
\Delta r \leq \frac{d_{k} d_{l}}{4 r_{0}}
$$

Equation (22) is identical to the range error equation (24.b) published in [15] derived for monostatic cases. On account of (3) the maximum phase error corresponding to maximum range error is finally given by

$$
\begin{aligned}
\Delta \phi & =\frac{2 \pi f_{c}}{\mathrm{c}} \max \{\Delta r\} \\
& =\frac{2 \pi f_{c}}{\mathrm{c}} \frac{d_{k}}{8 \cos \alpha}\left(\frac{d_{t}}{r_{t, 0}}+\frac{d_{r}}{r_{r, 0}}\right) .
\end{aligned}
$$

The phase error equation clearly states that beside the dependency on SAR geometry, the phase error caused by BiFBP is decided by the selection of subaperture lengths $d_{t}, d_{r}$ and subimage size $d_{k}$. It also depends strongly on the operating frequency of SAR systems.

BiFFBP [13] is another fast time-domain bistatic SAR algorithm. Similar to BiFBP, BiFFBP processes bistatic SAR echoes on a subaperture and subimage basis in BiFFBP. However, instead of processing the data in one beamforming stage and one backprojection stage, BiFFBP performs multiple beamforming stages before the backprojection stage. Equation (23) is also available for BiFFBP where $d_{k}, d_{t}$, and $d_{r}$ are the maximum dimension of the subimages, the transmitter subaperture length, and the receiver subaperture length, respectively, used in each beamforming stage. If the maximum dimension of the subimages decreases with a constant factor and

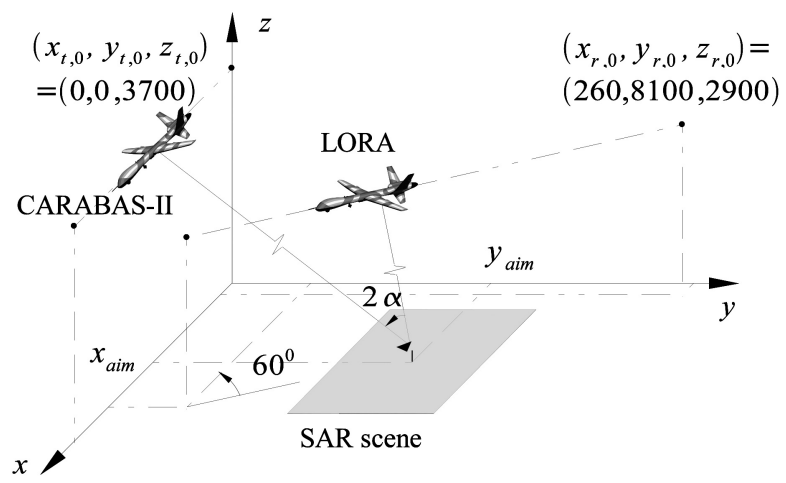

Fig. 5. SAR geometry utilized in simulations. Coordinates

$\left(x_{t, 0}, y_{t, 0}, z_{t, 0}\right)$ and $\left(x_{r, 0}, y_{r, 0}, z_{r, 0}\right)$ indicate initial positions of transmitter and receiver platforms. Angle formed by flight tracks of platforms is $60^{\circ}$.

the subaperture lengths increase with the same factor after every beamforming stage, the phase error will be unchanged.

\section{SIMULATION RESULTS AND VALIDATIONS}

In this section some simulation results are presented to validate the phase error equation (23) derived in the previous section. The simulated bistatic SAR system is mainly based on the parameters of CARABAS-II [16]. This means that the operating frequency range of the bistatic SAR system is decided by CARABAS-II. The transmitter is therefore assumed to be carried by this system. Some parameters of LORA [17] are utilized to simulate the platform carrying the passive receiver. The main parameters of CARABAS-II and LORA are summarized in Table I.

The ground SAR scene is simulated by a single point-like scatterer located at the aim point of the scene. Figure 5 sketches the SAR geometry. The initial positions of the transmitter and receiver platforms are determined by the coordinates $\left(x_{t, 0}, y_{t, 0}, z_{t, 0}\right)$ and $\left(x_{r, 0}, y_{r, 0}, z_{r, 0}\right)$. The flight tracks of two platforms create an angle of $60^{\circ}$. With this SAR geometry, the transmitter of the SAR system is on the side-looking mode while it is a mixing between forward-looking and down-looking modes for the receiver. Such SAR geometry is a challenge even for the time-domain algorithms like the extension of the fast backprojection algorithm introduced in [18] for bistatic cases.

According to the simulated SAR geometry, the angle $\alpha$ with respect to the aim point of the ground scene is maximized if the initial positions of the platforms are taken into account in the calculation of $\alpha$. With the information given in Table I and Fig. 5, this angle can be shown to be $\alpha \approx 45^{\circ}$. In this case (23) is rewritten by

$$
\Delta \phi=\frac{2 \pi f_{c}}{\mathrm{c}} \frac{d_{k}}{4 \sqrt{2}}\left(\frac{d_{t}}{r_{t, 0}}+\frac{d_{r}}{r_{r, 0}}\right)
$$


TABLE II

Maximum Phase Error Calculations with the System Parameters given in Table I

\begin{tabular}{c|ccccc}
\hline \hline$\Delta \phi$ & \multicolumn{5}{|c}{ Subaperture Length (aperture position) } \\
\hline Subimage (sample) & 16 & 32 & 64 & 128 & 256 \\
\hline $16 \times 16$ & $\pi / 64$ & $\pi / 32$ & $\pi / 16$ & $\pi / 8$ & $\pi / 4$ \\
$32 \times 32$ & $\pi / 32$ & $\pi / 16$ & $\pi / 8$ & $\pi / 4$ & $\pi / 2$ \\
$64 \times 64$ & $\pi / 16$ & $\pi / 8$ & $\pi / 4$ & $\pi / 2$ & $\pi$ \\
$128 \times 128$ & $\pi / 8$ & $\pi / 4$ & $\pi / 2$ & $\pi$ & $2 \pi$ \\
$256 \times 256$ & $\pi / 4$ & $\pi / 2$ & $\pi$ & $2 \pi$ & $4 \pi$ \\
\hline
\end{tabular}

where $r_{t, 0}$, and $r_{r, 0}$ are given in Table I, and $f_{c}$ can be determined from $f_{\max }$ and $f_{\min }$. The investigation into the phase error is now limited in the selections of $d_{k}$, $d_{t}$, and $d_{r}$. There always exists a trade-off between processing speed and phase error. A long subaperture and large subimage selection allows us to reduce the processing time of BiFBP and BiFFBP significantly though such selection may also lead to a very large phase error. Conversely, using a short subaperture and small subimage selection, the phase error can be minimized. However, the processing time requested by BiFBP and BiFFBP in this case increases. In this investigation, subimage size is chosen in the range $[16 \times 16,128 \times 128]$ samples with the image sample of $128 \times 128(\mathrm{~m} \times \mathrm{m})$ or $d_{k} \in[16 \sqrt{2}, 128 \sqrt{2}](\mathrm{m})$. For any choice, the mainlobe area of the scatterer's SAR image and its surrounding are ensured to be formed by four subimages. The center of the SAR image corresponds to the corners of these subimages. Hence, the maximum phase error always occurs in the center of the SAR image. The point selections of subaperture lengths are also limited in $[16,256]$ (aperture position) or $d_{t} \in[15,240](\mathrm{m})$ and $d_{r} \in[15.5,248](\mathrm{m})$. Table II summaries the approximate calculation results for the phase error using (24) with the above selections.

The far field condition in the antenna technique states that the effects caused by phase errors can be neglected if the phase error is smaller than or equal to $\pi / 8$ [19]. If we use this condition to limit the phase error, i.e., $\Delta \phi \leq \pi / 8$, the maximum subimage size is given $64 \mathrm{~m} \times 64 \mathrm{~m}$ or $d_{k}=64 \sqrt{2}(\mathrm{~m})$, while subapertures should not contain more than 128 positions or $d_{t}=120(\mathrm{~m})$ and $d_{r}=124(\mathrm{~m})$.

For evaluation purposes the SAR scene is reconstructed with BiGBP without phase errors, i.e., $\Delta \phi=0$. The contours of this reference SAR image are plotted in Fig. 6.

Figure 7 plots the contours of the reconstructed SAR scene in the range $[-30,0] \mathrm{dB}$ with a contour step of $-3 \mathrm{~dB}$. The figure shows BiFBP working with $d_{k}=32 \sqrt{2}(\mathrm{~m}), d_{t}=60(\mathrm{~m})$, and $d_{r}=62(\mathrm{~m})$. This selection corresponds to a maximum phase error of BiFBP $\Delta \phi \approx \pi / 8$. The contour plot shows clearly that the point-like scatterer is very well focused. The quality of the SAR image reconstructed with BiFBP is

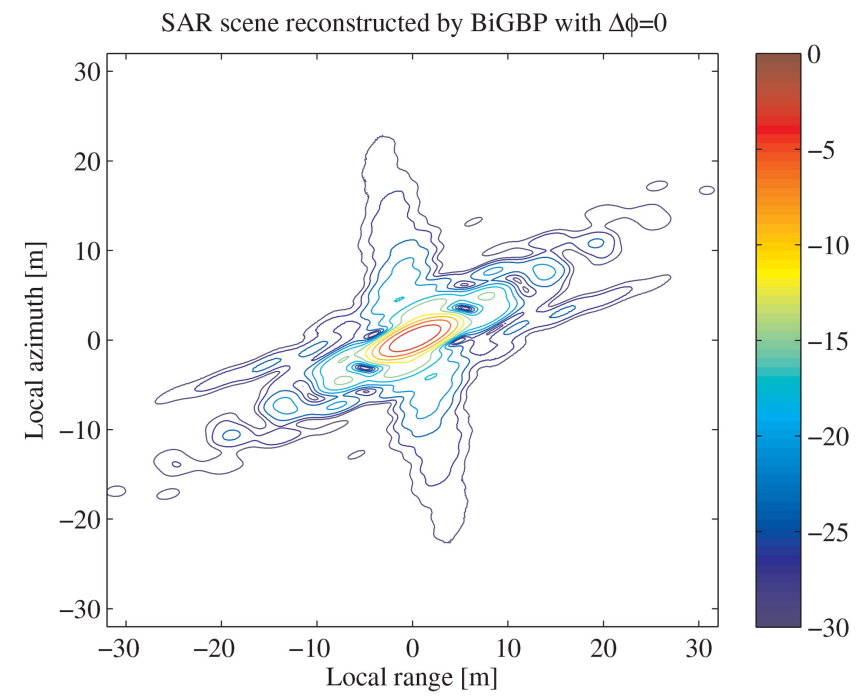

Fig. 6. SAR scene reconstructed by BiGBP with $\Delta \phi=0$.

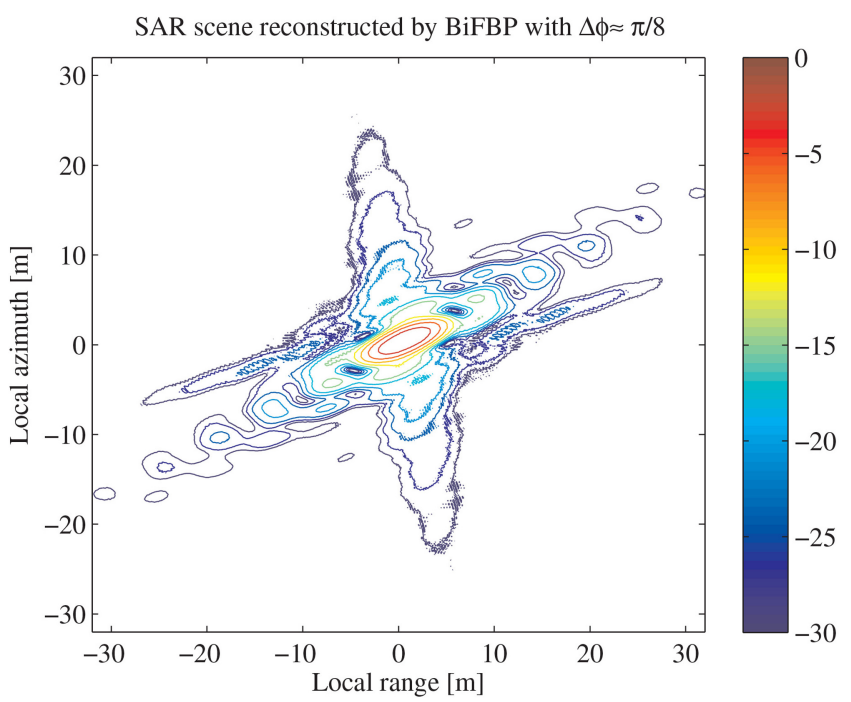

Fig. 7. SAR scene reconstructed by BiFBP with $\Delta \phi \approx \pi / 8$.

Center of scatterer's SAR image corresponds to corners of subimages. Phase error effect can clearly be observed in sidelobes.

slightly degraded in comparison to the reference SAR image given in Fig. 6 due to the phase error. However, those effects are invisible at the high contour levels $[-15,0] \mathrm{dB}$ and some small influences can clearly be observed at the lower contour levels $(\leq-18 \mathrm{~dB}$ or the cyan contours). In other words only sidelobes may suffer from the effects of the phase error in this specific case. This can be explained by the noise added due to the phase error. The added noise level is relatively small and thus does not strongly affect the peak signal level and its surrounding. However, the effects in certain directions (upper left and lower right) can be stronger in other directions (lower left and upper right). This phenomenon occurs due to the nonuniform distribution of the phase error, i.e., the phase error may be very large in certain directions, and depend strongly on the bistatic geometry. 


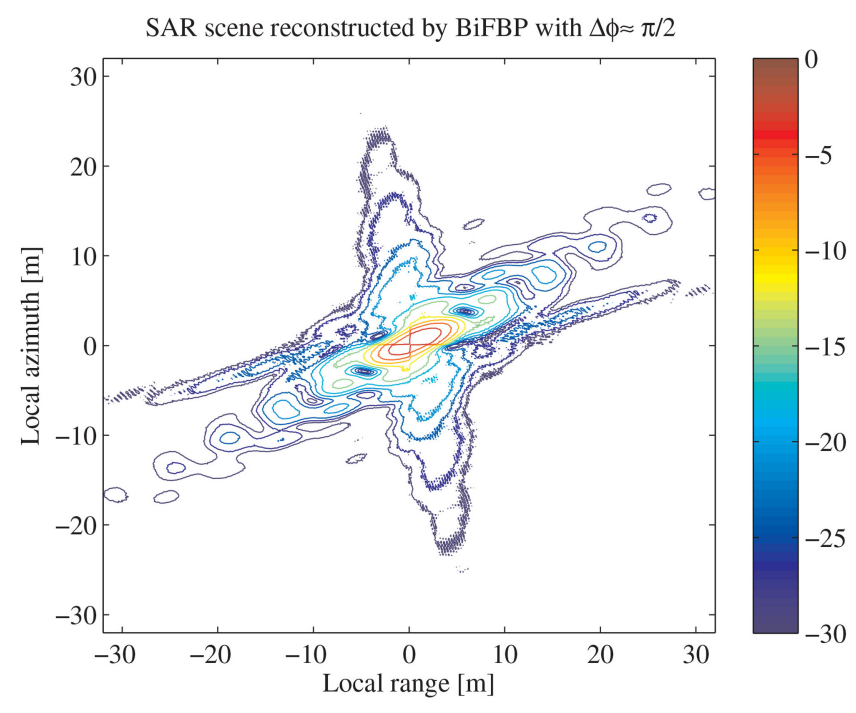

Fig. 8. SAR scene reconstructed by BiFBP with $\Delta \phi \approx \pi / 2$. Center of scatterer's SAR image corresponds to corners of subimages. Phase error effect can clearly be observed both in the mainlobe and in sidelobes. In mainlobe there is discontinuity between subimages and in sidelobes a higher noise level can be seen in comparison to Fig. 7.

Another selection of subapertures and subimages is again examined in which $d_{k}=64 \sqrt{2}(\mathrm{~m}), d_{t}=$ $120(\mathrm{~m})$, and $d_{r}=124(\mathrm{~m})$. The phase error caused by BiFBP with this selection is $\Delta \phi \approx \pi / 2$. The contour plot of the reconstructed SAR scene is given in Fig. 8. Please note that point target is placed in between subimages having maximum phase error. The effects of the phase error can even be observed at the $-3 \mathrm{~dB}$ contour level and by the cross in the middle of the SAR image. The mainlobe of the point-like scatterer is defocused and deformed. Both in the mainlobe and in the sidelobes, the effects of the phase errors are in some areas of the subimages more serious than in other areas. Such influences clearly show that the phase error degrades the quality of the SAR image significantly. The similar phenomenon caused by the ununiform distribution of the phase error can also be seen clearly in Fig. 8.

The evaluation has some limits since there is no definition on image quality measurements for bistatic SAR such as integrated sidelobe ratio (ISLR) and peak sidelobe ratio (PSLR). Resolution analysis of bistatic SAR is only sparsely investigated in [20]. Applying directly the currently used definitions like [21] cannot give correct measurement results. To have a more illustrative evaluation, the azimuth and range vectors at the centers of the SAR images are extracted and plotted in Fig. 9 and Fig. 10. As observed from these figures, the azimuth and range vectors in two cases $\Delta \phi=0$ and $\Delta \phi \approx \pi / 8$ are almost identical. However, there is a deformation of the azimuth and range vectors in the case $\Delta \phi \approx \pi / 2$ which can be observed in the figures. In the mainlobe areas of the azimuth and range vectors, there are the

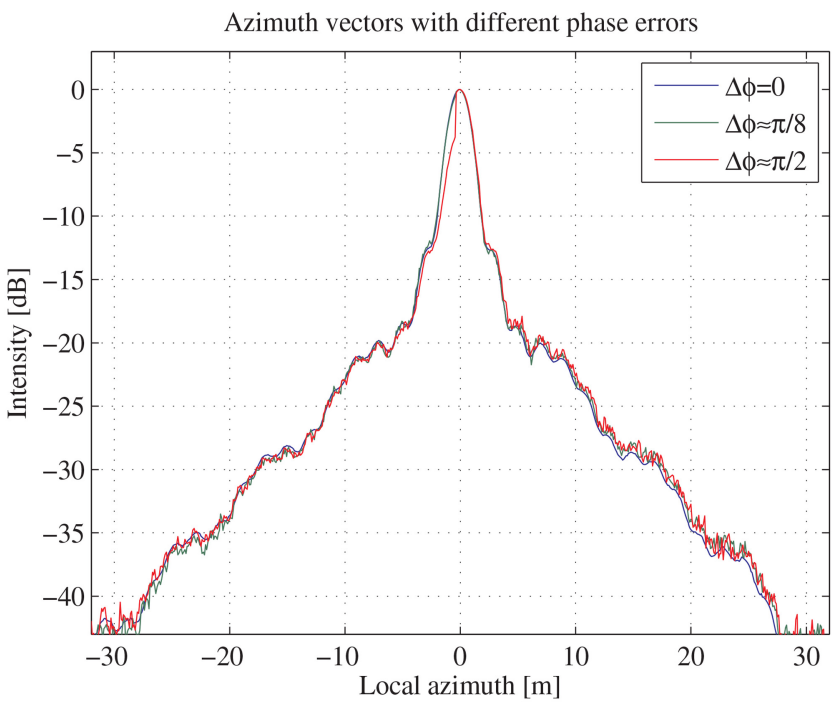

Fig. 9. Azimuth vectors with different phase errors. Phase error effects can clearly be observed in sidelobes for case of $\Delta \phi \approx \pi / 8$ and both in mainlobe and in sidelobes for case of $\Delta \phi \approx \pi / 2$.

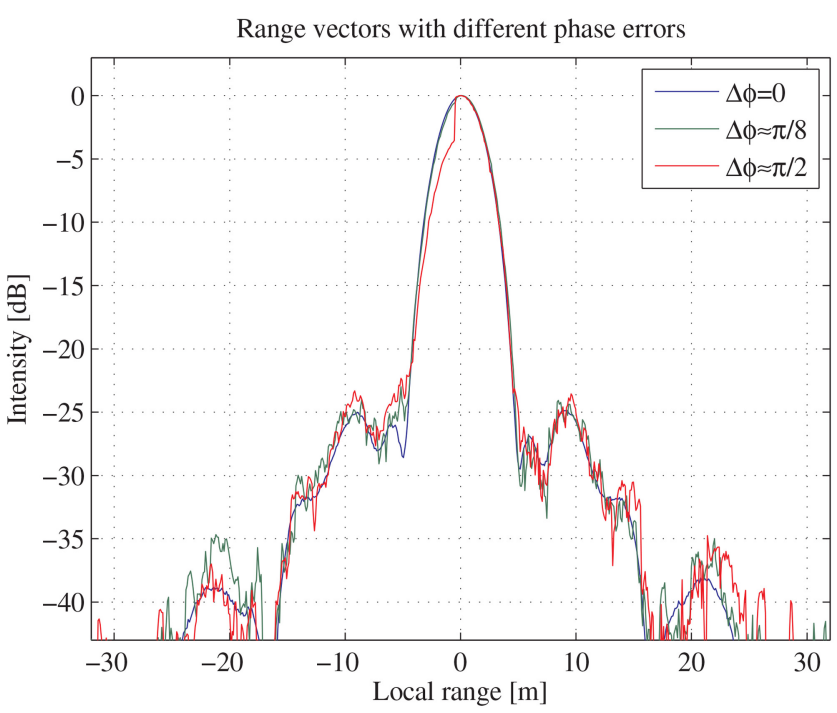

Fig. 10. Range vectors with different phase errors. Phase error effects can clearly be observed in sidelobes for case of $\Delta \phi \approx \pi / 8$ and both in mainlobe and in sidelobes for case of $\Delta \phi \approx \pi / 2$.

discontinuities on the right hand side of the plots. These discontinuities can also be observed in Fig. 8 at the $-3 \mathrm{~dB}$ level. The discontinuities are also the results of the ununiform distribution of the phase error related to the bistatic geometry.

\section{CONCLUSION}

In this paper the principle of calculating deterministic phase errors for bistatic time-domain SAR processing is given. This principle is utilized in the derivation of the phase error equation for the fast time-domain bistatic SAR algorithms BiFBP and BiFFBP. The derived phase error equation (23) is extremely crucial since it allows the estimation of the maximum phase errors caused by the bistatic 
algorithms with different algorithm parameters. In other words we can evaluate the trade-off between accuracy and speed of these algorithms. The derived phase error equation therefore helps to set the appropriate parameters for the algorithms. This equation is carefully examined and validated by some simulated results. In the simulations the parameters of CARABAS-II and LORA are used to build the bistatic SAR system.

\section{ACKNOWLEDGMENT}

The authors would like to thank the Swedish Defence Research Agency, Saab Bofors Dynamics, Saab Electronic Defence Systems, and RUAG Space for their cooperation.

VIET T. VU
THOMAS K. SJÖGREN
MATS I. PETTERSSON
Department of Electrical Engineering
Blekinge Institute of Technology
Campus Gräsvik
Karlskrona, 37143
Sweden
E-mail: (viet.thuy.vu@bth.se)

\section{REFERENCES}

[1] Qiu, X., et al.

A bistatic SAR raw data simulator based on inverse $\omega-k$ algorithm.

IEEE Transactions on Geoscience and Remote Sensing, 48, 3 (2010), 1540-1547.

[2] Rodriguez-Cassola, M., et al.

General processing approach for bistatic SAR systems: Description and performance analysis.

Proceedings of the 2010 8th IEEE European Conference on Synthetic Aperture Radar (EuSAR 2010), Aachen, Germany, June 2010, pp. 1-4.

[3] Ulander, L. M. H., et al.

Signal-to-clutter ratio enhancement in bistatic very high frequency (VHF)-band SAR images of truck vehicles in forested and urban terrain.

IET Radar, Sonar \& Navigation, 4, 3 (2010), 438-448.

[4] Walterscheid, I., et al.

Bistatic SAR experiments with PAMIR and

TerraSAR-X-Setup, processing, and image results.

IEEE Transactions on Geoscience and Remote Sensing, 48 , 8 (2010), 3268-3279.

[5] Rodriguez-Cassola, M., et al.

Bistatic TerraSAR-X/F-SAR spaceborne-airborne SAR experiment: Description, data processing, and results. IEEE Transactions on Geoscience and Remote Sensing, 48, 2 (2009), 781-794.

[6] Sanz-Marcos, J., et al.

SABRINA: A SAR bistatic receiver for interferometric applications.

IEEE Geoscience and Remote Sensing Letters, 4, 2 (2007), 307-311.

[7] Neo, Y. L., Wong, F. H., and Cumming, I. G

Processing of azimuth-invariant bistatic SAR data using the range Doppler algorithm.

IEEE Transactions on Geoscience and Remote Sensing, 46, 1 (2008), 14-21.

[8] Jun, S., Zhang, X., and Yang, J.

Principle and methods on bistatic SAR signal processing via time correlation.

IEEE Transactions on Geoscience and Remote Sensing, 46, 10 (2008), 3163-3178.
[9] Walterscheid, I., et al.

Bistatic SAR processing using an omega $-k$ type algorithm.

Proceedings of the 2005 IEEE International Geoscience and Remote Sensing Symposium (IGARSS'05), Seoul, Korea, July 2005, pp. 1064-1067.

[10] Qiu, X., Hu, D., and Ding, C.

An omega-k algorithm with phase error compensation for bistatic SAR of a translational invariant case.

IEEE Transactions on Geoscience and Remote Sensing, 46, 8 (2008), 2224-2232.

[11] Wong, F. H., Cumming, I. G., and Neo, Y. L.

Focusing bistatic SAR data using the nonlinear chirp scaling algorithm.

IEEE Transactions on Geoscience and Remote Sensing, 46, 9 (2008), 2493-2505.

[12] Vu, V. T., Sjögren, T. K., and Pettersson, M. I.

Fast backprojection algorithm for UWB bistatic SAR. Proceedings of the IEEE Radar Conference (RADAR), Kansas City, MO, May 2011, pp. 431-434.

[13] Vu, V. T., Sjögren, T. K., and Pettersson, M. I.

Fast factorized backprojection algorithm for UWB bistatic SAR image reconstruction.

Proceedings of the 2011 IEEE International Geoscience and Remote Sensing Symposium (IGARSS'2011),

Vancouver, Canada, July 2011, pp. 4237-4240.

[14] Ulander, L. M. H., et al.

Fast factorized back-projection for bistatic SAR processing.

Proceedings of the 2010 8th European Conference on Synthetic Aperture Radar (EUSAR), Aachen, Germany, June 2010, pp. 1-4.

[15] Ulander, L. M. H., Hellsten, H., and Stenstrom, G. Synthetic-aperture radar processing using fast factorized back-projection.

IEEE Transactions on Aerospace and Electronic Systems, 39, 3 (2003), 760-776.

[16] Gustavsson, A., et al.

Development and operation of an airborne VHF SAR system-Lessons learned.

Proceedings of the 1998 IEEE International Geoscience and Remote Sensing Symposium (IGARSS'98), vol. 1, Seattle, WA, July 1998, pp. 458-462.

[17] Ulander, L. M. H. and Hellsten, H.

Low-frequency ultrawideband array-antenna SAR for stationary and moving target imaging.

Proceedings of SPIE, Radar Sensor Technology IV, vol. 3704, Orlando, FL, Apr. 1999, pp. 149-158.

[18] Yegulalp, A. F.

Fast backprojection algorithm for synthetic aperture radar. Record of the 1999 IEEE Radar Conference, Waltham, MA, Apr. 1999, pp. 60-65.

[19] Balanis, C. A.

Antenna Theory Analysis and Design (3rd ed.). Hoboken, NJ: Wiley, 2005.

[20] Garza, G. and Qiao, Z.

Resolution analysis of bistatic SAR.

Proceedings of SPIE, Radar Sensor Technology XV, vol. 8021, Orlando, FL, Apr. 2011, pp. 80211V-1-80211V-6.

[21] $\mathrm{Vu}, \mathrm{V}$. T., et al.

Definition on SAR image quality measurements for UWB SAR.

Proceedings of SPIE, Image and Signal Processing for Remote Sensing XIV, vol. 7109, Cardiff, UK, Sept. 2008, pp. 71091A-1-71091A-9. 